\title{
EFFECTS OF THE PRESENCE OF SUPERCRITICAL WINDS
}

\author{
Michael Friedjung \\ Institut d'Astrophysique \\ 98 bis Boulevard Arago, 75014 Paris, France.
}

\begin{abstract}
Evidence is given that classical novae have optically thick winds for a certain time after optical maximum. Multifrequency observations especially of FH Ser indicate acceleration of the wind at very large optical depths, probably by radiation pressure of an object above the Eddington limit. This result though fairly convincing needs checking by proper radiative transfer calculations. A way in which it might be produced is mentioned as is the influence of the wind on the structure of the ejected envelope.
\end{abstract}

\section{Introduction}

Inspite of the fact that novae have been known and studied for a long time, one is struck by the fact that many basic features are very badly understood. This is particularly true for the interpretation of the development of a nova after optical maximum. Features are observed especially in the spectrum, which do not seem to belong to the same world as that of present day theories! To quote Shara (1989) "A detailed quantitative understanding and simulation of outburst spectra may require an effort comparable to that invested by theorists over the last two decades in simulating thermonuclear runaways". It is clear that much physics is needed in such investigations. I hope that this talk will make a useful contribution to such an aim.

It should be emphasized that nova outbursts involve violent non stationary phenomena, so there is a possibility of effects being seen, which are not present in less "abnormal" stars. Certain processes may not be hindered in novae, and we should not have too many prejudices!

In this short review I shall examine the properties derivable from observation of the strong winds, which appear to be present for some time after optical maximum. The post optical maximum evolution of novae is very probably determined by the interaction between these winds. Reasons will be given for supposing not only that these winds are optically thick, but also for supposing them accelerated by radiation pressure acting on the absorption of the continuous spectrum. This can happen if the luminosity is above the Eddington limit, and arguments will also be given indicating that this occurs. Possible mechanisms for producing such a situation which is normally difficult to produce will be mentioned, as well as effects due to the interaction between different winds. 


\section{General nature of ejection by novae}

There are good reasons for believing the old idea (Whipple and Payne-Gaposchkin 1936) of continuing ejection by novae after optical maximum ( see for example Friedjung 1977). This is based both on the properties of the line and of the continuous spectrum.

The first sort of reasoning follows from the profiles of spectrum lines. They indicate the presence of layers expanding outwards with different velocities. After optical maximum higher velocity material usually appears later, suggesting that its ejection is later. Such material also tends to be more ionized, suggesting greater closeness to a source of ionizing radiation if photoionization dominates. In addition McLaughlin (1947) gave more direct reasons for believing that material in deeper layers nearer the centre of the envelope tends normally to have higher velocities. McLauglin's reasoning is based on what happens when lines blend, and emission or absorption from material in a certain layer belonging to a line, is at the same wavelength as emission or absorption of another layer in another line. Line emission from an outer layer will not be absorbed by line absorption of an inner layer, while line emission of an inner layer will be absorbed by line absorption of an outer layer. Another reason given by McLaughlin is that the parts of spectrum line profiles due to higher velocity material vary more during the relatively rapid light variations over time scales of days, which are sometimes seen. Let us finally note that high velocity material can produce strong absorption components in profiles, indicating production of at least much of the radiation of the continuous spectrum in even deeper layers.

McLaughlin's reasoning clearly assumes spherical symmetry, but the fact that the same type of velocity stratification is indicated by many different arguments makes it difficult to deny the conclusion. It follows that as usually no stationary or nearly stationary layers are seen near the centre of the envelope, they would if present need to be hidden by a large optical depth in the continuous spectrum. This suggests that acceleration of the wind must be conceived as occuring at large optical depths, at least at times when line absorption due to the high velocity material is seen. In this respect a nova is very different from a more "normal" star. It may be noted that HR Del before its December 1967 maximum may be an exception which does not contradict the rule for novae after optical maximum, as fairly narrow emission lines were then seen, which could have come from central regions.

Another type on reasoning is based on multifrequency observations of the total luminosity and of the continuous specrum. While the total radiative luminosity does not decrease by a large amount in the early stages after optical maximum, the general wavelength distribution of the continuous spectrum with its peak, shifts to higher energies (though oscillations may sometimes be superposed on this general variation). If the radiation is considered as being emitted by an optically thick photosphere, its temperature therefore appears to increase and its radius to decrease with time. In view of what has been deduced from line profiles, one might expect such a photosphere to be produced by expanding material, that is by an optically thick wind. In this framework the decrease of photospheric radius can be interpreted as indicating that in later stages the wind is only optically thick to smaller radii, that is the mass loss rate is lower.

Several types of optically thick wind are possible, and criteria need to be given in order to decide which occur for individual classical novae. In what follows I shall 
suppose that the properties of the wind are related to those of the continuous spectrum, whose radiation comes from optical depths of the order of unity, and that the velocities of such layers at a given time are those of the highest velocity material which contributes in the formation of line profiles.

The nature of winds such as those we are dealing with, is indicated by the values of the various forms of energy flux (radiative, kinetic, etc.) and by the ratios of the densities of different forms of energy in the wind. Considering the latter first, one can see why the ratios are important if the equation of motion of a fluid is examined without taking account of viscosity or possible effects of magnetic fields. One form of this equation for a spherically symmetric steady state wind is:

$$
d / d r E_{K}=d / d r\left(E_{M}-2 E_{G} / 3-E_{R} / I\right)
$$

Here $\mathrm{r}$ is the radial distance from the centre of the wind, $\mathrm{E}_{K}$ the kinetic energy density, $\mathrm{E}_{M}$ the gravitational energy density (ie potential energy due to gravitation per unit volume), $\mathrm{E}_{G}$ the gas thermal energy density, $\mathrm{E}_{R}$ the radiation energy density and $\mathrm{I}$ a factor equal to 3 for isotropic radiation. For acceleration to occur the sum of the pressure terms in $\mathrm{E}_{G}$ and $\mathrm{E}_{R}$ must be larger than that in $\mathrm{E}_{M}$. When $\mathrm{E}_{R}$ is larger than $\mathrm{E}_{G}$, acceleration is mainly by radiation pressure. Moreover it is clear that significant acceleration can only occur in regions where the term in $\mathrm{E}_{K}$ is not much larger than the sum of the other terms. Integrating eq. (1) this means that significant acceleration to the terminal velocity cannot occur at $r$ 's where $E_{K}$ is much larger than both $E_{G}$ and $\mathrm{E}_{R}$. If this condition is not satisfied in parts of the wind which can be directly observed, one might expect it to be satisfied in unobservable parts, which could at small $r$ 's and large optical depths. In such a case one should be able to determine the characteristics of the region where the wind is accelerated, by extrapolation from observable regions.

When wind acceleration is mainly by radiation pressure, the variation of this pressure with $\mathrm{r}$ depends on what sort of opacity is dominant. Winds of "normal" O stars are now thought to be accelerated by the action of radiation pressure in spectrum lines in outer regions. Naturally this kind of mechanism cannot work in such regions if the value of $\mathrm{E}_{R}$ there is much less than that of $\mathrm{E}_{K}$. In that situation radiation pressure in the continuum may still be effective at large optical depths, where line opacity can be negligible. In the last case a luminosity above the Eddington limit is required, which is that for electron scattering when that is the dominant opacity. It must be noted that the relevant luminosity is that at the base of an optically thick wind; part will be converted to gravitational potential energy and part to kinetic energy. Therefore a sufficient condition for the presence of a super critical (super Eddington) wind is for the sum of the fluxes of radiation and kinetic energy in observable regions of a wind to be above the Eddington limit.

The estimate of the total radiative flux has become much more reliable in recent years because of the availability of multifrequency obsevations, which I shall use in what follows. There is naturally still some uncertainty due to errors in the distance determinations of novae. To obtain the other quantities required I shall assume the result of Bath (1978) that opacity due to electron scattering dominates in the continuum of optically thick winds having effective temperatures above $6000 \mathrm{~K}$ and lumunosities between 0.5 and 2.0 of the Eddington luminosity. When hydrogen is completely ionized 
(effective temperatures over $9000 \mathrm{~K}$ ) the radius of the "scattersphere" (surface of last electron scattering here taken at an electron scattering optical depth of $P$ ) is given by:

$$
R_{s}=P / \kappa_{s c} \rho_{s}
$$

In eq. (2) $\rho_{s}$ is the density of the scattersphere and $\kappa_{s c}$ the constant electron scattering opacity. The radiative energy density just above the scattersphere will then be estimated as equal to the total radiative flux divided by $4 \pi \mathrm{c} \mathrm{R}_{s}{ }^{2}$, with $\mathrm{c}$ the velocity of light. The kinetic energy flux is given by:

$$
F_{K}=2 \pi P R_{s} V_{s}^{3} / \kappa_{s c}
$$

with $V_{s}$ the ejection velocity near the scattersphere. The kinetic energy density near the scattersphere is then the kinetic energy flux divided by $4 \pi V_{s} R_{s}{ }^{2}$, and the ratio of kinetic energy density to radiative energy density is the flux ratio multiplied by $\mathrm{V}_{s} / \mathrm{c}$, clearly independent of $R_{s}$. Let us also note that the gas thermal energy density in the scattersphere is $1.210^{8} \rho_{s} \mathrm{~T}_{s} / \mu$, with $\mathrm{T}_{s}$ the scattersphere temperature and $\mu$ the mean molecular weight. The ratio $\mathrm{E}_{K} / \mathrm{E}_{G}$ just above the scattersphere equals $410^{-9} \mathrm{~V}_{s}^{2}$ $\mu / \mathrm{T}_{s}$.

At large optical depths In an optically very thick wind with an opacity dominated by electron scattering, as long as the velocity in a layer is not very different from that in the scattersphere:

$$
E_{K} / E_{R}=0.25\left(F_{K} / F_{R}\right)\left(V_{s} / c\right)\left(r / R_{s}\right)
$$

and

$$
E_{K} / E_{G}=410^{-9} V_{s}^{2} \mu / T
$$

with $\mathrm{T}$ the local temperature.

It is not easy to directly estimate $R_{s}$ from observations of the continuous spectrum, and in fact information about radiative transfer in an optically thick wind is required. Radiation will be emitted by a photosphere of radius $\mathrm{R}_{p}$, which for an optically thick wind with a continuous opacity dominated by electron scattering, will in general be less than $R_{s}$. In the absence of good models I shall assume Planckian emission at a temperature $\mathrm{T}_{p}$ by a sphere of radius $\mathrm{R}_{p}$, as long as the observed spectral distribution of radiation emitted is close to that of a black body. It may be noted in this connection that the models of Harkness (1983) for optically thick winds appear to be unsuitable (Andrade and Friedjung 1989) for various reasons, including especially the assumption of solar abundances and the lack of a full non LTE treatment. Putting things in terms of $\mathrm{R}_{p}$, we can to a first approximation replace eq. (3) by:

$$
F_{K}=2 \pi Q R_{p} V_{s}^{3} / \kappa
$$

Here $\kappa$ equals $\left(\kappa_{s c} \kappa_{a b s}\right)^{0.5}$, with $\kappa_{a b s}$ the continuous pure absorption opacity, while $\mathrm{P}$ is replaced by $Q$. The wind velocity is assumed to be the same at $R_{p}$ and $R_{s}$. Comparing eqs. (3) and (6) we may note that $R_{s} / R_{p}=Q \kappa_{s c} / P \kappa$.

The calculations of Bath (1978) even changing his assumed solar hydrogen abundance suggest that $\kappa$ is generally less than 0.15 , so in the following calculations what should be a minimum $F_{K}$ will be estimated from eq. (6) taking this value of $\kappa$ and 
a probably too small value for $Q$ of $2 / 3 . F_{K}$ will also be underestimated because $V_{s}$ derived from the radial velocity of observed spectral line absorption components formed in the wind, is the average projected radial velocity in the line of sight, and so less than the wind velocity.

\section{Application to particular novae}

Let us now apply the previous general considerations to particular novae, so as to test the nature of ejection for them.

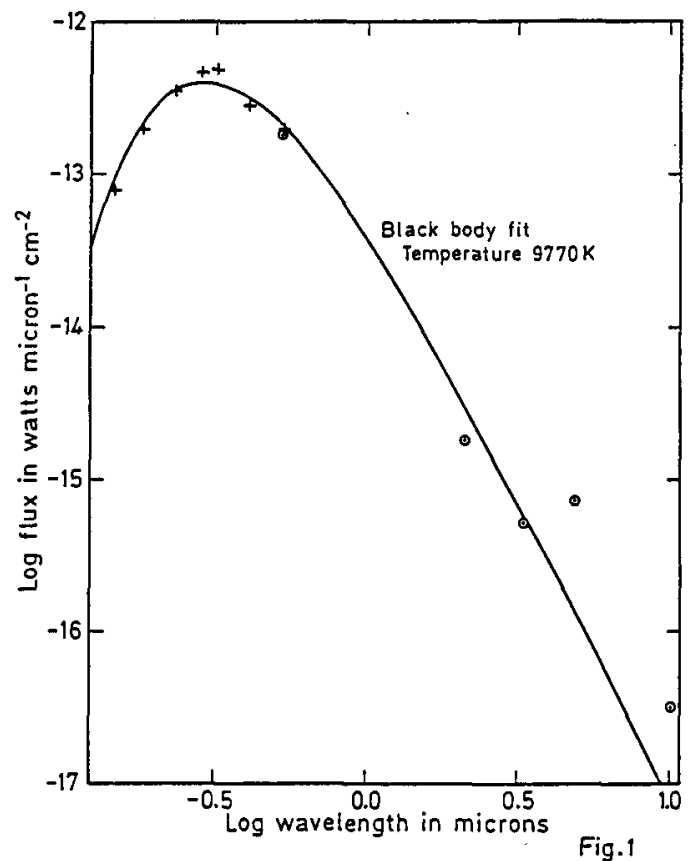

Energy distribution of FH Ser Mareh 131970

(a) FH Ser 1970

This was the first nova to be observed in the satellite ultraviolet, while broad band photometry was also performed in the infrared. One can therefore obtain information about the spectral distribution of radiation emitted over a wide wavelength range on dates for which there are both ultraviolet photometric data, and infrared data obtained either at a similar time, or which can be interpolated between observations for two other dates. Such broad band data for March 131970 about a month after the initial outburst are shown in Figure 1 using the infrared data of Geisel et al (1970) (circles) in addition to the ultraviolet data of Gallagher and Code (1974) (crosses), obtained by the satellite OAO-2. One can see from this figure that the observations are fairly well fitted by a Planckian energy distribution over a wavelength range of about twenty to 
one. Only the two points furthest in the infrared show a moderate excess with respect to the fit. It was on such a basis that in previous writings on this nova I made a Planckian energy distribution assumption, in order to obtain the total luminosity and $T_{p}$, from which $R_{p}$ could be obtained. In Friedjung (1987a) infrared data of Geisel et al were added to those of Gallagher and Code for all dates from 22.05 days (except for 49.83 days) after Febuary 141970 at $0.0 \mathrm{~h}$. The values of $F_{K}$ given in that paper are what I have here described as probably minimum values. According to that paper the radiative luminosity was not quite constant but declined somewhat, while the sum of this luminosity and $\mathrm{F}_{K}$ stayed well above the Eddington limit for some time. However it must be noted that the calculated value of the radiative luminosity is proportional to the square of the assumed distance ( $650 \mathrm{pc}$ in the calculations) and $F_{K}$ to the assumed distance itself. The assumed interstellar reddening is important, while the calculated $F_{K}$ depends also on other assumptions. Nevertheless errors due to such assumptions would have to be rather large to in particular change the conclusion about the sum of luminosities.

The minimum energy density ratios $\mathrm{E}_{K} / \mathrm{E}_{R}$ determined near the photosphere lie between 300 and 1200 , while values of $\mathrm{E}_{K} / \mathrm{E}_{G}$ are all of the order of $110^{4} \mu$ (if as previously assumed for the region just above the scattersphere, most radiation also came from one direction in the region now considered). One can estimate that $\mathrm{E}_{R}$ should have been of the order of $E_{K}$ at $r / R p$ not more than $310^{-3}-10^{-2}$, while $E_{G}$ should have been smaller (by a factor of $10^{-1}-10^{-2}$ ) there. This suggests that most acceleration would have been produced at such radii by radiation rather than by gas pressure. In these acceleration regions at very large optical depths radiation pressure in lines might be expected not to be all that important, so support is again given to the presence of a super Eddington luminosity, as already indicated by the measurements of the total luminosity. The present argument depends on gas pressure being small, which it must have been unless $\mathrm{F}_{K}$ was even much larger than estimated in the last paragraph.

\section{(b) $\mathrm{V} 1668$ Cyg 1978}

This nova was observed in various spectral regions, and in particular in the ultraviolet by the satellite IUE in its first year. Unfortunately the descriptions of the continuous spectrum by Stickland et al $(1979,1981)$ are not very detailed. They succeeeded in fitting the energy distribution to a black body, and calculated the variation of the total radiative luminosity with time. They found a decrease by a factor of about three between 2 and 9 days after optical maximum, followed by a constant luminosity phase to 23 days after optical maximum. The authors did not like the idea of a super Eddington luminosity, so they reduced the first distance determination to $2.2 \mathrm{kpc}$, in order to have a maximum bolometric luminosity at the Eddington limit. This is clearly not necessary in the framework of what I am saying ! If we are rather conservative and suppose that on September 23.971978 about 20 days after optical maximum the total radiative flux was only $110^{38} \mathrm{ergs}^{-1}$, and estimate from the maximum of the published energy distribution a $T_{p}$ of $11600 \mathrm{~K}$, an $\mathrm{R}_{p}$ of $310^{12} \mathrm{~cm}$ is found. The minimum value of $F_{K}$ is then $810^{38}$, and the sum of the two fluxes still appears to be clearly above the Eddington limit. The value found for the ratio $\mathrm{E}_{K} / \mathrm{E}_{R}$ near the photosphere is 1100 , again suggesting acceleration at large optical depths as for FH Ser. 


\section{(c) Other Novae}

Full analyses of the continuous spectra of other novae have not yet been carried out, and it is premature to draw conclusions concerning them. However total radiative luminosities have been measured for the novae V1370 Aql (1982) and GQ Mus (1983). The data of Snijders et al (1987) for V1370 Aql suggest that if it was at a distance of $5 \mathrm{kpc}$, there was a plateau in the luminosity variation about 50 days after outburst at $1.510^{38} \mathrm{ergs} \mathrm{s}^{-1}$, not far below the Eddington limit. Taking a distance of $4.8 \mathrm{kpc}$ for GQ Mus, Krautter et al (1984) found that the radiative luminosity of this nova three days after discovery was probably 1.5 times the Eddington luminosity, and about half the Eddington luminosity six weeks later. If as suggested for the novae considered previously, the minimum kinetic energy flux was greater than that of radiation which escaped, the total luminosity of these two novae would appear to have been well above the Eddington limit at the times quoted here.

\section{Discussion}

The arguments given suggest the presence of optically thick winds accelerated by radiation pressure. Such a point of view was not only previously suggested by me (Friedjung 1966), but also by Bath and Shaviv (1976). The hydrodynamics of such winds was studied by Ruggles and Bath (1979), while Kato $(1983,1985)$ also studied similar optically thick winds. It must be emphasised that what is suggested is continued ejection; this is not the same situation as that thought to exist for supernovae, which are considered to eject optically thick envelopes very rapidly. A comparison can also be made with the theory of Wolf Rayet star winds given by Turolla et al (1988); in this theory, though radiation pressure acting in spectral lines is important, there is significant acceleration at fairly large electron scattering optical depths but only little below the thermalization radius. However as we have seen the acceleration of nova winds would appear to be at depths where probably there is thermalization.

The conclusions drawn here clearly depend on the interpretatrion of the observations. It is the analysis of nova spectra in particular which indicates the presence of optically thick winds. Following this we can use multifrequency observations to find the total radiative luminosity; the uncertainties in distance and interstellar absorption produce perhaps in general more uncertainties than the extrapolation to unobserved spectral regions. The estimated radiative flux is proportional to the square of the estimated distance, while the estimated kinetic energy flux is proportional to the estimated distance. The determination of the kinetic energy flux is clearly affected by the uncertainties in the determination of $\mathrm{R}_{p}$; these depend on assumptions about radiative transfer, spherical symmetry and the validity of supposing that $T_{p}$ exists, as well as the relation of such a quantity to the observed dependence of emitted radiation on wavelength. Therefore though present arguments appear quite strong, one needs especially to do proper radiative transfer calcultions for optically thick winds before one can be really certain.

One can next ask whether other observed properties can be explained in the present framework. Bath and Shaviv explained an observed correlation of properies of novae 
with brightness during the development after optical maximum, by an almost constant radiative luminosity near the Eddington limit in this stage. However the usual apparent correlation of wind velocity with $\mathrm{R}_{p}$ cannot be so explained. If the winds were like those of normal hot stars, one might expect a relation between the wind velocity and the escape velocity near a stationary photosphere. As the radius of this photosphere decreased the wind velocity would then be expected to increase, as is observed. Even if such a model could be assumed, the observations of FH Ser would indicate rather large values of the ratio of the wind velocity to the escape velocity (of 16 or more from 15 to 31 days after Feb 14 1970, if the mass of the central object is $1.5 \mathrm{M}_{O}$ corresponding to a typical total mass for the binary which would still have to be present below the stationary photosphere). In the framework of the ideas supported in this talk the explanation should be rather different. In work I did some years ago (Friedjung 1981) I tried to use the Ruggles and Bath (1979) theory to find the properties of the region where an optically thick wind could be accelerated by radiation pressure acting on continuous absorption. It was considered that most acceleration would occur where the fluxes of diffusive radiation and advected radiaion were of the same order. For luminosities far above the Eddington limit the flux of radiation leaving and of kinetic energy should then be of the same order. An order of magnitude condition for the velocity is obtained in this situation:

$$
F_{R} / R_{p}=100 V_{s}^{3}
$$

where $\mathrm{F}_{R}$ is the total radiative flux leaving the nova. When a comparison is made with the observations of FH Ser the velocity predicted is too small, the agreement being better if the factor 100 is replaced by 10 . The theory is clearly still not good enough.

In any case if what I have been saying is at least basically correct, we still need to know how a relatively steady wind accelerated by a super Eddington luminosity is possible. One explanation which requires serious consideration is that the extra luminosity which brings the total above the Eddington limit is due to the interaction of the companion of the binary with the envelope of the expanded white dwarf, in which the companion orbits during the early development of a nova after optical maximum. This type of theory has already been considered by MacDonald (1980) and by MacDonald et al (1985), but much remains to be done. Deviations from spherical symmetry are certainly very important then. It also remains to be seen whether enough energy can be pumped into the wind, and whether this would work for novae like GK Per, which has a longer period and therefore a larger orbit than other novae. It is only if and when such problems are sorted out that one can try to make reliable predictions about the time variation of the velocity of the wind.

It may be noted that the expected deviations from spherical symmetry might produce a dependence of the properties of novae during their oubursts obseved from the earth, on the inclinations of the orbits of these binaries to the line of sight. I made an attempt to look for such a dependence last year (1988), but found no clear evidence for any effect, perhaps because observed properties should also depend on many other factors, which are difficult to disentangle.

Other effects can be expected in the presence of strong winds, which are worth briefly recalling, though they are outside the field of the title of this talk. In a paper (Friedjung 1987b) I considered what would be the result of interaction of the wind with material ejected around the start of the outburst, that is with material belonging to 
what is called the premaximum system. A thin dense shell would be formed, which could end up by containing most of the mass of the envelope, and which might be where spectrum line components of the principal system are formed. A hot plasma would be expected to be created inside the shell; $X$ ray and coronal line emission from it might be fairly weak and so hard to detect. Nova spectra however also show greater complexity such as the presence sometimes of very many absorption components of spectrum lines and the doubling of the absorption components belonging to the principal system. The former of these could be due to instabilities leading to the formation of cool clouds in the hot plasma, while the latter taking up an old idea, might be due to the radiation pressure of Lyman $\alpha$ photons acting inside the shell. Such ideas as well as a number of others proposed particularly by Soviet workers, need to be tested.

Much remains to be done to study the phenomena described in this talk. The field should offer many possibilities for a lot of work and very lively argument!

\section{References}

Andrade, A. A., Friedjung, M.: 1989, Astron. Astrophys. in press.

Bath, G. T.: 1978, Mon. Not. R. astr. Soc. 182, 35.

Bath, G. T., Shaviv, G.: 1976, Mon. Not. R. astr. Soc. 175, 305.

Friedjung, M.: 1966, Mon. Not. R. astr. Soc. 132, 317.

Friedjung, M. 1977, in "Novae and Related Stars", ed M. Friedjung, Reidel, Dordrecht, p. 61 .

Friedjung, M.: 1981, Acta Astron. 31, 373.

Friedjung, M.: 1987a, Astron. Astrophys. 179, 164.

Friedjung, M.: 1987b, Astron. Astrophys. 180, 155.

Gallagher, J.S., Code, A.D.: 1974, Astrophys. J. 189, 303.

Geisel, S. L., Kleinmann, D. E., Low, F. J.: 1970, Astrophys. J. 161, L101.

Harkness, R. P.: 1983, Mon. Not. R. astr. Soc. $204,45$.

Kato, M.: 1983, Publ. astr. Soc Japan $\underline{35}, 507$.

Kato, M.: 1985, Publ. astr. Soc. Japan $\underline{37}, 19$.

Krautter, J., Beuermann, K., Leitherer, C., Oliva, E., Moorwood, A. F. M., Deul, E., Wargau, W., Klare, G., Kohoutek, L., van Paradijs, J., Wolf, B.: 1984, Astron. Astrophys. 137, 307.

MacDonald, J.: 1980, Mon. Not. R. astr. Soc. 191, 933.

MacDonald, J., Fujimoto, M. Y., Truran, J. W.: 1985, Astrophys. J. 294, 263.

McLaughlin, D. B.: 1947, Publ. astr. Soc. Pacific $\underline{59}, 244$.

Ruggles, L. L. N., Bath, G. T.: 1979, Astron. Astrophys. 무, 97.

Shara, M.: 1989, Publ. astr. Soc. Pacific 101, 5.

Snijders, M. A. J., Batt,T. J., Roche, P. F., Seaton, M. J., Morton, D. C., Spoelstra, T. A. T., Blades, J. C.: 1987, Mon. Not. R. astr. Soc. 228, 329.

Stickland, D., Penn, C. J. Seaton, M. J., Snijders, M. A. J., Storey, P. J., Kitchin, C.R.: 1979, in "The First Year of IUE", ed. A. J. Willis, University College, London, p. 63.

Stickland, D. J., Penn, C. J., Seaton, M. J., Snijders, M. A. J., Storey, P.J.: 1981. Mon. Not. R. astr. Soc. $197,107$.

Turolla, R., Nobili, L., Calvani, M.: 1988, Astrophys. J. $\underline{324}, 899$.

Whipple, F. L., Payne-Gaposchkin, C.: 1936, Circ astr. Obs. Harvard 413. 